\title{
Association between the Arylalkylamine N-Acetyltransferase $(A A N A T)$ Gene and Seasonality in Patients with Bipolar Disorder
}

\author{
So Yung Yang ${ }^{1,2}$, Kyung Sue Hong ${ }^{3,4}$, Youngah $\mathrm{Cho}^{3}$, Eun-Young $\mathrm{Cho}^{4}$, Yujin $\mathrm{Choi}^{4}$, \\ Yongkang $\mathrm{Kim}^{5}$, Taesung Park ${ }^{5}$, Kyooseob Ha ${ }^{6,7}$, and Ji Hyun Baek ${ }^{3 凶}$ \\ ${ }^{1}$ Department of Psychiatry, National Health Insurance Service Ilsan Hospital, Goyang, Republic of Korea \\ ${ }^{2}$ Institute of Behavioral and Science in Medicine, Yonsei University College of Medicine, Seoul, Republic of Korea \\ ${ }^{3}$ Department of Psychiatry, Sungkyunkwan University School of Medicine, Samsung Medical Center, Seoul, Republic of Korea \\ ${ }^{4}$ Center for Clinical Research, Samsung Biomedical Research Institute, Seoul, Republic of Korea \\ ${ }^{5}$ Department of Statistics, Seoul National University, Seoul, Republic of Korea \\ ${ }^{6}$ Department of Psychiatry, Seoul National University College of Medicine, Seoul, Republic of Korea \\ ${ }^{7}$ Institute of Human Behavioral Medicine, Seoul National University College of Medicine, Seoul, Republic of Korea
}

Objective Bipolar disorder (BD) is complex genetic disorder. Therefore, approaches using clinical phenotypes such as biological rhythm disruption could be an alternative. In this study, we explored the relationship between melatonin pathway genes with circadian and seasonal rhythms of BD.

Methods We recruited clinically stable patients with BD $(\mathrm{n}=324)$. We measured the seasonal variation of mood and behavior (seasonality), and circadian preference, on a lifetime basis. We analyzed 34 variants in four genes (MTNR1a, MTNR1b, AANAT, ASMT) involved in the melatonin pathway.

Results Four variants were nominally associated with seasonality and circadian preference. After multiple test corrections, the rs116879618 in $A A N A T$ remained significantly associated with seasonality (corrected $\mathrm{p}=0.0151$ ). When analyzing additional variants of $A A N A T$ through imputation, the rs117849139, rs77121614 and rs28936679 (corrected p=0.0086, 0.0154, and 0.0092) also showed a significant association with seasonality.

Conclusion This is the first study reporting the relationship between variants of AANAT and seasonality in patients with BD. Since AANAT controls the level of melatonin production in accordance with light and darkness, this study suggests that melatonin may be involved in the pathogenesis of $\mathrm{BD}$, which frequently shows a seasonality of behaviors and symptom manifestations.

Psychiatry Investig 2021;18(5):453-462

Key Words Bipolar disorder, Seasonality, Circadian rhythm, Melatonin pathway, AANAT.

\section{INTRODUCTION}

Bipolar disorder (BD) is chronic psychiatric disorder characterized by episodic changes in mood and energy level. ${ }^{1} \mathrm{BD}$ has a high heritability, close to $80 \% .{ }^{2,3}$ However, because of the heterogeneity of the biological basis and complexity of genetic architecture, there have been limited findings in genetic stud-

Received: December 17, 2020 Revised: January 24, 2021

Accepted: March 4, 2021

$\bowtie$ Correspondence: Ji Hyun Baek, MD, PhD

Department of Psychiatry, Sungkyunkwan University School of Medicine, Samsung Medical Center, 81 Irwon-ro, Gangnam-gu, Seoul 06351, Republic of Korea

Tel: +82-2-3410-3000, Fax: +82-2-3410-0050, E-mail: jihyunbaek@skku.edu (c) This is an Open Access article distributed under the terms of the Creative Commons Attribution Non-Commercial License (https://creativecommons.org/licenses/bync/4.0) which permits unrestricted non-commercial use, distribution, and reproduction in any medium, provided the original work is properly cited. ies. ${ }^{4,5}$ Therefore, alternative approaches adopting specific clinical features and biological markers as phenotypes for genetic studies were suggested..$^{6-8}$

Organisms have evolved innate biological clocks that oscillate with the environmental cycles of day and night (circadian rhythm) and photoperiod change (seasonal rhythm) for adaptation. ${ }^{9-11}$ This process is also important for humans to adjust to external cues, ${ }^{12,13}$ but abnormally exaggerated or dampened rhythmic behavioral changes have been observed in some mental disorders. ${ }^{1,15} \mathrm{In} \mathrm{BD}$, the variation of biological rhythms, such as seasonal mood, behavioral changes, or circadian rhythm abnormalities have been suggested as distinctive clinical characteristics. ${ }^{16-18}$ Although abnormalities in biological rhythms are more prominent during episodes, they are also identified in euthymic state $\mathrm{BD}$ and regarded as lifetime traits. A seasonal 
pattern is common in $\mathrm{BD}$, even among treated patients whose seasonal fluctuations may have been modified by such treatment. In addition, BD who not experienced either seasonal manic or depressive episodes, also reported to have higher scores for seasonal variation in mood and behavior when compared to the general population or patients with depression. ${ }^{19,20} \mathrm{Cli}-$ matic conditions may trigger BD symptoms or episodes ${ }^{21-23}$ and antimanic drugs have been known to stabilize circadian rhythms. ${ }^{24,25}$ Also, BD with seasonal depressive episodes or evening preference have been reported to be associated with other specific clinical profiles, e.g., bipolar II disorder subtype, comorbid eating disorders and premenstrual syndrome, more relapses and rapid cycling. ${ }^{18,26-29}$

Melatonin is a neurohormone secreted from the pineal gland during the hours of darkness. It falls rapidly with light onset. Arylalkylamine $\mathrm{N}$-acetyltransferase (AANAT) is a key regulatory enzyme in the melatonin biosynthesis pathway, which converts serotonin to $\mathrm{N}$-acetylserotonin. $\mathrm{N}$-acetylserotonin is converted to melatonin by acetylserotonin O-methyltransferase (ASMT).${ }^{30}$ Oscillating levels of activated AANAT result in the rhythmic synthesis and secretion of melatonin. ${ }^{31}$ Seasonal photoperiod-induced changes in melatonin secretion have widereaching effects on seasonal animal physiology and behavior, as the MT1 and MT2 melatonin receptors (encoded by MTN$R 1 a$ and MTNR1b) are distributed widely throughout the body, including the central nervous system, heart, endocrine system, and immune system. ${ }^{11,32}$

Considering the abnormal biological rhythms and melatonin levels observed in patients with $\mathrm{BD}^{17,18,33}$ and the role of melatonin in diurnal and seasonal changes, ${ }^{11,32}$ melatonin seems to be related to the biological mechanisms that develops the clinical characteristics of the subgroup of BD. Genetic studies for $\mathrm{BD}$ focusing on genes in the melatonin pathway reported mixed results. ${ }^{33-36}$ A genetic heterogeneity of $\mathrm{BD}$ may be one reasons for this discrepancy. There have also been association studies using biological rhythm disruption as an alternative phenotype in BD. Geoffroy et al. ${ }^{37}$ reported a negative finding in their investigation of the association between seasonal depressive episodes and the circadian genes in the melatonin pathway. Other study regarding sleep patterns of $\mathrm{BD}$ and healthy controls showed an association between the ASMT variant and the inter-day stability of sleep. ${ }^{38}$

We hypothesized that patients with BD with abnormal biological rhythms as a lifetime trait are likely to have a specific genetic underpinning, especially related to the melatonin pathway. This study investigated the association between the melatonin pathway genes, the circadian preferences, and seasonal mood and behavior changes in $\mathrm{BD}$.

\section{METHODS}

\section{Subjects}

We recruited clinically stable patients who met the DSMIV diagnostic criteria for BD $(\mathrm{n}=324)$, including bipolar I disorder $(\mathrm{n}=182)$, bipolar II disorder $(\mathrm{n}=134)$ and BD not otherwise specified $(n=8)$ between 18 and 60 years of age from the outpatient and inpatient units of the Samsung Medical Center $(n=148)$ and the Seoul National University Bundang Hospital $(n=176)$ in South Korea. Board-certified psychiatrists who had at least one year of research experience examined the participants' psychiatric diagnoses using the DSM-IV criteria with either the Structured Clinical Interview for DSM-IV Axis I Disorders (SCID) ${ }^{39}$ or the Korean version of the Diagnostic Interview for Genetic Studies (DIGS).$^{40}$ SCID was used at Seoul National University Bundang Hospital and DIGS was used at the Samsung Medical Center. No significant difference in terms of demographic characteristics was detected between participants evaluated using SCID and DIGS. We excluded participants if they could not clearly remember their lifetime traits due to illnesses. Moreover, to prevent the effect of current mood state or medication in evaluating their lifetime characteristics, we included participants after their medication had been stabilized. All participants were under the standard pharmacological treatment, including mood stabilizers (lithium, valproate, lamotrigine, and carbamazepine) or atypical antipsychotics (quetiapine, olanzapine, risperidone, aripiprazole, and ziprasidone). We excluded those with mental retardation, substance abuse, medical illnesses, or long-term use of hormonal agents known to affect mood. Because sustained social rhythm can deeply affect the biological rhythm, ${ }^{41}$ we also excluded nightshift workers as participants. We obtained written informed consent from all subjects after a complete explanation of the study. This study was approved by the Institutional Review Boards of the Samsung Medical Center (IRB No. 2012-09-056) and the Seoul National University Bundang Hospital (IRB No. B-1105/128-008).

\section{Measurements of phenotypes}

We measured circadian preference by using the standardized Korean version of the Composite Scale of Morningness (CSM), ${ }^{42,43}$ containing 13 questions concerning what time participants preferred to go to bed, wake up, and perform specific activities. The sum of the answers to these questions yielded a single score to represent the level of circadian preference (range, 13-55), with lower scores indicating a stronger evening preference. We measured seasonality with Seasonal Pattern Assessment Questionnaire (SPAQ) ${ }^{44}$ containing 6 items to measure seasonal variations in sleep, social activity, mood, weight, appetite, and energy level. The Korean version of the SPAQ that 
was translated into Korean was used with the permission of the original author. The translation and validation process for this scale has been described in our previous study. ${ }^{45}$ We used the sum of individual item scores, each on a 5-point scale ranging from 0 (no change) to 4 (extremely marked change) to indicate a global seasonality score (GSS). Based on the criteria of Kasper et al. ${ }^{46,47}$ for seasonal affective disorder (SAD) and sub- syndromal SAD, and a GSS of 9 or greater, we designated scores with a subjective rating of having at least mild difficulty with seasonal changes [on a 6-point scale from 0 point (no difficulty) to 5 points (a disabling difficulty); 1 points=mild] or a GSS of 11 or higher as having significant seasonality. We assigned both seasonality and circadian preference on a lifetime-basis.

Table 1. Characteristics of SNPs in melatonin pathway genes

\begin{tabular}{|c|c|c|c|c|c|c|c|}
\hline Gene & SNP & Genomic location* & Intragenic location & M & $\mathrm{m}$ & MAF & HWF \\
\hline MTNR1a & rs28611030 & Chr4:187448441 & 3'-near & $\mathrm{A}$ & G & 0.213 & 0.867 \\
\hline MTNR1a & rs7440284 & Chr4:187449434 & 3'-near & $\mathrm{C}$ & $\mathrm{T}$ & 0.039 & 1.000 \\
\hline MTNR1a & rs1800884 & Chr4:187455426 & Exon 3 & G & A & 0.011 & 1.000 \\
\hline MTNR1a & rs34532313 & Chr4:187460490 & Intron 1 & $\mathrm{~T}$ & $\mathrm{C}$ & 0.475 & 1.000 \\
\hline MTNR1a & rs116952947 & Chr4:187463252 & Intron 1 & $\mathrm{C}$ & $\mathrm{T}$ & 0.028 & 1.000 \\
\hline MTNR1a & rs6820205 & Chr4:187464867 & Intron 1 & $\mathrm{C}$ & $\mathrm{T}$ & 0.092 & 0.315 \\
\hline MTNR1a & rs13140444 & Chr4:187465729 & Intron 1 & $\mathrm{C}$ & $\mathrm{T}$ & 0.406 & 0.908 \\
\hline MTNR1a & rs149982127 & Chr4:187472823 & Intron 1 & $\mathrm{~T}$ & $\mathrm{C}$ & 0.017 & 1.000 \\
\hline MTNR1a & rs4862706 & Chr4:187473694 & Intron 1 & A & G & 0.088 & 0.292 \\
\hline MTNR1a & rs1800885 & Chr4:187476360 & Exon 1 & G & A & 0.039 & 1.000 \\
\hline MTNR1a & rs76691596 & Chr4:187479828 & 5'-near & G & $\mathrm{T}$ & 0.056 & 0.066 \\
\hline MTNR1a & rs6858707 & Chr4:187484762 & 5 -near & G & A & 0.386 & 0.724 \\
\hline MTNR1a & rs13131052 & Chr4:187485933 & 5'-near & $\mathrm{T}$ & G & 0.196 & 0.110 \\
\hline MTNR1b & rs75715438 & Chr11:92699831 & 5 'near & $\mathrm{T}$ & $\mathrm{C}$ & 0.025 & 1.000 \\
\hline MTNR1b & rs75153006 & Chr11:92700218 & 5 'near & G & $\mathrm{T}$ & 0.037 & 0.357 \\
\hline MTNR1b & rs4753426 & Chr11:92701596 & 5 'near & $\mathrm{C}$ & $\mathrm{T}$ & 0.313 & 0.437 \\
\hline MTNR1b & rs10830963 & Chr11:92708710 & Intron 1 & $\mathrm{C}$ & G & 0.428 & 0.733 \\
\hline MTNR1b & rs3781637 & Chr11:92713770 & Intron 2 & $\mathrm{~T}$ & $\mathrm{C}$ & 0.146 & 0.826 \\
\hline MTNR1b & rs148736119 & Chr11:92714049 & Intron 2 & G & A & 0.020 & 1.000 \\
\hline MTNR1b & rs76309303 & Chr11:92717504 & Intron 3 & G & A & 0.014 & 1.000 \\
\hline MTNR1b & rs1447350 & Chr11:92718127 & Exon 4 (3'-UTR) & $\mathrm{C}$ & G & 0.315 & 0.607 \\
\hline MTNR1b & rs12225378 & Chr11:92718649 & Exon 4 (3'-UTR) & $\mathrm{T}$ & $\mathrm{C}$ & 0.079 & 0.434 \\
\hline MTNR1b & rs1447352 & Chr11:92722761 & 3'-near & A & G & 0.315 & 0.605 \\
\hline$A A N A T$ & rs495055 & Chr17:74440890 & 5'-near & $\mathrm{A}$ & G & 0.071 & 1.000 \\
\hline AANAT & rs9896887 & Chr17:74444059 & 5'-near & G & A & 0.495 & 0.823 \\
\hline AANAT & rs116879618 & Chr17:74448849 & 5 'near & $\mathrm{C}$ & $\mathrm{T}$ & 0.042 & 0.434 \\
\hline AANAT & rs77537806 & Chr17:74449204 & 5'-near & G & $\mathrm{T}$ & 0.022 & 1.000 \\
\hline AANAT & rs3744044 & Chr17:74475014 & 3'-near & $\mathrm{C}$ & $\mathrm{T}$ & 0.011 & 1.000 \\
\hline$A S M T$ & rs17149149 & ChrX:1734143 & Exon 2 & $\mathrm{C}$ & A & 0.020 & 1.000 \\
\hline$A S M T$ & rs62593301 & ChrX:1743087 & Intron 3 & $\mathrm{~T}$ & $\mathrm{C}$ & 0.195 & 0.374 \\
\hline$A S M T$ & rs28675287 & ChrX:1748910 & Intron 6 & $\mathrm{~T}$ & C & 0.346 & 0.112 \\
\hline$A S M T$ & rs4521942 & ChrX:1752017 & Intron 7 & G & $\mathrm{T}$ & 0.059 & 0.297 \\
\hline$A S M T$ & rs4639690 & ChrX:1755236 & Intron 8 & G & A & 0.353 & 0.542 \\
\hline$A S M T$ & rs4933063 & ChrX:1755404 & Exon 9 & $\mathrm{C}$ & $\mathrm{T}$ & 0.311 & 1.000 \\
\hline
\end{tabular}

${ }^{*}$ Genomic Location was released from Human Feb. 2009 (GRCh37/hg19) Assembly. Chr: chromosome, SNP: single nucleotide polymorphism, M: major allele, m: minor allele, MAF: minor allele frequency, HWE: p-value of the Hardy-Weinberg equilibrium test, UTR: untranslated region 


\section{SNP selection and genotyping}

We isolated a genomic DNA sample from peripheral blood leukocytes by using the Wizard Genomic DNA Purification Kit (Promega, Madison, WI, USA), according to the manufacturer's instructions. We produced the genotype data by using the Korea Biobank Array Chip (K-CHIP), version 1.0 from the $\mathrm{K}-\mathrm{CHIP}$ consortium. The K-CHIP, consisting of about $833 \mathrm{~K}$ SNPs for the whole genome, was designed by the Center for Genome Science, Korea National Institute of Health, Korea (4845-301, 3000-3031). According to the Affymetrix Axiom ${ }^{\circledR}$ 2.0 Assay User Guide, the K-CHIP assay was conducted by Axiom $^{\circledR}$ 2.0 Reagent Kit (Affymetrix, Santa Clara, CA, USA), which contains a series of reactions including amplification, fragmentation, hybridization, and ligation. After ligation, we stained the reaction product, imaged and analyzed it for genotype reading by using the Genotyping Console ${ }^{\mathrm{TM}}$ Software (Affymetrix, Santa Clara, CA, USA). We produced the genotype data for approximately 790K SNPs after having checked quality control for the samples.

We selected four melatonin related genes (MTNR1a, MTNR1b, AANAT, ASMT). We explored single nucleotide polymorphisms (SNPs) within 10 kilobase pairs upstream and downstream from the coding and regulatory region of each of the genes. The former three genes are located in autosomes, and the ASMT is located in the pseudoautosomal region 1 (PAR1) of sex chromosomes. It had previously been suggested that these are linked to $\mathrm{BD} .^{48}$ We included 34 SNPs with minor allele frequencies (MAF) greater than $1 \%$, and genotype call rates $>97 \%$ with the associated analyses. Table 1 summarizes the localization of the studied SNPs. To confirm the reliability of the genotyping method using the K-CHIP, we analyzed all SNPs with cluster plots. Four selected SNPs (rs34532313 on MTNR1a, rs10830963 on MTNR1b, rs9896887 on AANAT, and rs4639690 on ASMT) that showed the highest MAF for each gene were re-genotyped for 48 random samples by sequencing reaction using ABI PRISM ${ }^{\circledR}$ BigDye Terminator v 3.1 Cycle Sequencing Kits (Applied Biosystems, Foster City, CA, USA). The concordance rate of genotype data between sequencing and assay using the array Chip was $99.5 \%$.

\section{Statistical analyses}

We checked the Hardy-Weinberg equilibrium with the Fisher's exact test for genotype analysis. We observed no significant deviation in any of these SNPs (Table 1). We evaluated the genotype associations between the selected SNPs and each phenotype by logistic and linear regression analysis, with age and sex as covariates. We considered the additive genetic models based on the minor alleles of each SNP. We controlled for experimental type I errors by using Bonferroni correction, covering all included SNPs (corrected $\mathrm{p}=0.05 * \mathrm{SNP}$ number). We did the statistical analyses with the R program, v.3.2.2 (R Foundation for Statistical Computing, Vienna, Austria) and PLINK, v. 1.9 (www.cog-genomics.org/plink/1.9). ${ }^{49}$

We imputed genes that have a significant variant in the above process by using the reference panel of the EAS data from the 1000 Genomes Project Integrated Phase 3 Release. Likewise, we included SNPs within 10 kilobase pairs upstream and downstream from each gene. The data was phased using SHAPEIT $(\mathrm{v} 2.837)^{50}$ and imputed using IMPUTE2 (2.3.0). We selected variants with a high imputation quality ('info' score $>0.5$ ). ${ }^{51} \mathrm{We}$ subsequently analyzed imputed SNPs with minor allele frequencies (MAF) greater than $1 \%$, and genotype call rates $>97 \%$ for association by using PLINK v.1.9. We used the Haploview software $4.0^{52}$ to estimate and plot pairwise linkage disequilibrium (LD) measures of SNPs that were included in associated analyses in this study. The LD blocks were defined according to Gabriel criteria. ${ }^{53}$

\section{RESULTS}

Demographic and clinical characteristics of participants $(\mathrm{n}=$ $324)$ were demonstrated in Table 2 . About $38 \%(n=123)$ of the participants presented with seasonality and $25 \%(n=83)$ presented with evening preference based on the cut-off value derived from the Korean general population. ${ }^{43}$ Patient with seasonality demonstrated much younger age $(\mathrm{p}=0.04)$, earlier age of onset $(\mathrm{p}=0.02)$ and no difference in bipolar I or II subtypes. The earlier onset age in $\mathrm{BD}$ with seasonality was consistent with other previous studies (Table 3). ${ }^{26,37}$

Table 2. Demographic and clinical characteristics of subjects $(\mathrm{N}=324)$

\begin{tabular}{lc}
\hline \multicolumn{1}{c}{ Characteristics } \\
\hline Sex, male, N (\%) & $111(34.26)$ \\
Age, years, mean (SD) & $34.94(10.49)$ \\
Education, college graduate or more, N (\%) & $214(66.25)$ \\
Marital state, married, N (\%) & $144(44.58)$ \\
Occupation, present, N (\%) & $263(81.17)$ \\
GSS, mean (SD) & $7.45(5.29)$ \\
Seasonality*, present, N (\%) & $123(38.32)$ \\
CSM score, mean (SD) & $31.06(7.76)$ \\
Evening preference, present, N (\%) & $83(25.61)$ \\
Age at onset, years, mean (SD) & $23.89(8.75)$ \\
Duration of illness, years, mean (SD) & $11.79(7.53)$ \\
\hline
\end{tabular}

*the significant seasonality was defined by having either seasonal affective disorder or subsyndromal seasonal affective disorder according to the definition of Kasper et al., ${ }^{46}$ the lower CSM score indicates a stronger evening preference. SD: standard deviation, GSS: global seasonality score, CSM: composite scale of morningness 
Table 3. Differential clinical characteristics of patients with or without seasonality

\begin{tabular}{lccc}
\hline & & Seasonality* & \multicolumn{1}{c}{$\mathrm{p}$} \\
\cline { 2 - 4 } & $(-)(\mathrm{N}=198)$ & $(+)(\mathrm{N}=123)$ & 0.522 \\
Sex, male, N (\%) & $71(35.9)$ & $39(31.7)$ & 0.040 \\
Age, mean (SD) & $35.0(26.0 ; 44.0)$ & $80(65.0)$ & 0.810 \\
Education, college graduate or more, N (\%) & $132(67.0)$ & $99(80.5)$ & 0.971 \\
Occupation, present, N (\%) & $161(81.3)$ & $54(44.3)$ & 0.997 \\
Marrital state, married (\%) & $89(44.9)$ & $66(53.7)$ & 0.407 \\
Subtype, type I, N (\%) & $114(57.6)$ & $20.0[16.0 ; 27.0]$ & 0.020 \\
Age at onset, mean (SD) & $22.0[18.0 ; 30.0]$ & $9.0[6.0 ; 17.0]$ & 0.678 \\
Duration of illness, mean (SD) & $11.0[6.0 ; 16.0]$ & $30.2 \pm 8.1$ & 0.133 \\
CSM_score, mean (SD) & $31.6 \pm 7.6$ & \\
\hline
\end{tabular}

*the significant seasonality was defined by having either seasonal affective disorder or subsyndromal seasonal affective disorder by the definition from Kasper et $\mathrm{al}^{46}$ This statistic excluded patients with missing values (N=3). SD: standard deviation

Single marker analysis identified nominal associations (uncorrected $\mathrm{p}<0.05)$ between seasonality and three SNPs in melatonin pathway genes. These were rs116879618 located in AANAT ( $\mathrm{p}=0.0004), \mathrm{rs} 1800885$ in MTNR1a $(\mathrm{p}=0.031)$, and rs4639690 in ASMT ( $\mathrm{p}=0.0497)$. After adjusting for multiple testing by using the Bonferroni correction, the association remained significant for rs116879618 in AANAT (corrected $\mathrm{p}=$ 0.0151 ). GSS showed nominal associations with rs 116879618 located in AANAT ( $\mathrm{p}=0.0199)$, but it did not remain significant after multiple test corrections. Circadian preference showed a nominal association (uncorrected $\mathrm{p}<0.05$ ) with $\mathrm{rs} 75715438$ in MTNR1b ( $\mathrm{p}=0.0144$ ), but it did not remain significant after adjusting multiple testing (Table 4). All analyses data are shown in Supplementary Table 1 (in the online-only Data Supplement).

Since AANAT showed significant association with $\mathrm{BD}$, we performed additional imputation analysis. We generated the genotypes of 20 additional SNPs through imputation following quality control, and we conducted an associated analysis for each SNP and seasonality. Figure 1 presents the locations of the AANAT SNPs and their linkage disequilibrium block. An additional five SNPs showed a nominal association, and three SNPs, rs117849139, rs77121614, and rs28936679 (corrected $\mathrm{p}=0.0086,0.0154$, and 0.0092 ) showed a significance association following multiple test corrections. Among these SNPs, rs28936679 is a nonsynonymous (missense) variant in exon 1 (Table 5). Supplementary Table 2 (in the online-only Data Supplement) presents detailed information about the imputed SNPs and the statistical data.

\section{DISCUSSION}

The aim of this study was to evaluate whether the melatonin pathway genes are associated with biological rhythm changes in patients with $\mathrm{BD}$. Assuming that specific clinical presentations have a more homogeneous genetic underpinning, we evaluated the genetic association of phenotypes reflecting the circadian and seasonal rhythms with the melatonin pathway genes as functional candidate genes.

In a previous study, $\mathrm{BD}$ patients demonstrated a hypersensitive pineal response to ocular light exposure when compared to a control, independent of the disease state. ${ }^{54}$ Bipolar I disorder showed a lower baseline melatonin level and a lower nadir melatonin level on the light night and a greater amplitude of variation in melatonin secretion than did the controls on the dark night. ${ }^{55}$ These studies and the result of the current study support the possibility that the melatonin pathway genes are involved in the exaggerated biological rhythm changes in $\mathrm{BD}$.

Four variants in AANAT, ASMT, MTNR1a, and MTNR1b have been nominally associated with seasonality and circadian preference. Following correction for multiple testing, the rs116879618 in the AANAT remained significantly associated with the seasonality of $\mathrm{BD}$. In past studies, genomewide linkage analyses with bipolar families have shown that the $17 \mathrm{q} 25$ region, including the $A A N A T$, is linked to $\mathrm{BD},{ }^{56-58}$ and associated analyses with the bipolar population shows polymorphism of AANAT being associated with BD. ${ }^{34}$ But some studies failed to find a significant result. ${ }^{35,36}$ The rs 116879618 , which appeared to be significantly associated with the seasonality of $\mathrm{BD}$ in the current study, has not been reported to be significantly associated with $\mathrm{BD}$ or any other psychiatric disorders. Its clinical significance was not well defined, but it is located in 5'-UTR of the AANAT and may influence the regulatory function of this region. The AANAT has been labeled the "timezyme" ${ }^{\text {"59 }}$ because of its role in the timing of melatonin production by the pineal gland along with light and darkness. Activity increases 10 to 100 fold at night, causing an increase in the production and the release of melatonin, and in response to light, it shows rap- 
Table 4. Summary of association analysis of SNPs in melatonin pathway genes and seasonality and circadian preference of patients with bipolar disorder

\begin{tabular}{|c|c|c|c|c|c|c|c|c|c|c|}
\hline \multirow{2}{*}{ Gene } & \multirow{2}{*}{ SNP } & \multicolumn{3}{|c|}{ Global seasonality score } & \multicolumn{3}{|c|}{ Seasonality } & \multicolumn{3}{|c|}{ CSM score } \\
\hline & & Beta & SE & $\mathrm{p}^{\dagger}$ & OR & CI & $\mathrm{p}^{\dagger}$ & Beta & SE & $\mathrm{p}^{\dagger}$ \\
\hline MTNR1a & rs28611030 & -0.183 & 0.506 & 0.718 & 0.933 & $0.628-1.385$ & 0.729 & -0.294 & 0.709 & 0.678 \\
\hline MTNR1a & rs7440284 & 1.310 & 1.096 & 0.233 & 2.161 & $0.942-4.961$ & 0.069 & -0.782 & 1.529 & 0.610 \\
\hline MTNR1a & rs1800884 & -0.867 & 2.011 & 0.667 & 0.649 & $0.122-3.444$ & 0.612 & 1.883 & 2.754 & 0.495 \\
\hline MTNR1a & rs 34532313 & -0.400 & 0.418 & 0.340 & 0.974 & $0.704-1.346$ & 0.872 & 0.809 & 0.578 & 0.163 \\
\hline MTNR1a & rs116952947 & -0.740 & 1.319 & 0.575 & 1.266 & $0.463-3.463$ & 0.647 & 1.015 & 1.803 & 0.574 \\
\hline MTNR1a & rs6820205 & -0.024 & 0.707 & 0.973 & 1.206 & $0.701-2.075$ & 0.499 & 1.712 & 0.960 & 0.076 \\
\hline MTNR1a & rs13140444 & -0.210 & 0.426 & 0.623 & 0.930 & $0.669-1.293$ & 0.666 & 0.718 & 0.588 & 0.223 \\
\hline MTNR1a & rs149982127 & -0.603 & 1.621 & 0.710 & 0.677 & $0.173-2.647$ & 0.575 & 0.283 & 2.305 & 0.902 \\
\hline MTNR1a & rs4862706 & -0.083 & 0.711 & 0.907 & 1.151 & $0.667-1.986$ & 0.613 & 1.347 & 0.973 & 0.167 \\
\hline MTNR1a & rs1800885 & 1.631 & 1.092 & 0.136 & 2.522 & $1.088-5.847$ & 0.031 & -0.100 & 1.534 & 0.948 \\
\hline MTNR1a & rs76691596 & -0.974 & 0.853 & 0.255 & 1.049 & $0.544-2.025$ & 0.886 & 2.006 & 1.161 & 0.085 \\
\hline MTNR1a & rs6858707 & -0.356 & 0.436 & 0.414 & 0.817 & $0.581-1.149$ & 0.245 & 0.166 & 0.605 & 0.783 \\
\hline MTNR1a & rs13131052 & -0.372 & 0.501 & 0.458 & 1.002 & $0.680-1.477$ & 0.991 & 0.598 & 0.691 & 0.387 \\
\hline MTNR1b & rs75715438 & -0.868 & 1.349 & 0.520 & 0.775 & $0.260-2.313$ & 0.647 & 4.513 & 1.834 & 0.014 \\
\hline MTNR1b & rs75153006 & -0.300 & 1.069 & 0.779 & 0.962 & $0.419-2.210$ & 0.927 & -2.809 & 1.458 & 0.055 \\
\hline MTNR1b & rs4753426 & 0.268 & 0.439 & 0.542 & 1.046 & $0.743-1.473$ & 0.795 & 0.698 & 0.611 & 0.255 \\
\hline MTNR1b & rs10830963 & -0.560 & 0.412 & 0.175 & 0.820 & $0.594-1.134$ & 0.230 & 0.113 & 0.573 & 0.844 \\
\hline MTNR1b & rs3781637 & 0.011 & 0.595 & 0.985 & 1.150 & $0.729-1.813$ & 0.548 & 0.987 & 0.818 & 0.228 \\
\hline$M T N R 1 b$ & rs148736119 & -1.685 & 1.468 & 0.252 & 0.446 & $0.118-1.686$ & 0.234 & -2.785 & 2.118 & 0.190 \\
\hline$M T N R 1 b$ & rs76309303 & 0.373 & 1.888 & 0.843 & 1.714 & $0.418-7.034$ & 0.454 & 0.492 & 2.586 & 0.849 \\
\hline MTNR1b & rs 1447350 & 0.316 & 0.441 & 0.474 & 1.073 & $0.764-1.508$ & 0.685 & 0.927 & 0.604 & 0.126 \\
\hline MTNR1b & rs12225378 & 1.401 & 0.753 & 0.064 & 1.332 & $0.748-2.370$ & 0.330 & 1.630 & 1.044 & 0.120 \\
\hline MTNR1b & rs1447352 & 0.330 & 0.440 & 0.454 & 1.069 & $0.761-1.502$ & 0.700 & 0.907 & 0.607 & 0.136 \\
\hline$A A N A T$ & rs495055 & -0.906 & 0.823 & 0.272 & 0.682 & $0.349-1.335$ & 0.264 & -1.189 & 1.130 & 0.294 \\
\hline AANAT & rs9896887 & 0.668 & 0.422 & 0.115 & 1.207 & $0.870-1.677$ & 0.260 & -0.843 & 0.579 & 0.147 \\
\hline AANAT & rs116879618 & 2.368 & 1.012 & 0.020 & 5.034 & $2.043-12.410$ & $<0.001^{*}$ & -1.040 & 1.390 & 0.455 \\
\hline$A A N A T$ & rs77537806 & 1.533 & 1.444 & 0.289 & 2.419 & $0.801-7.304$ & 0.117 & 0.463 & 1.987 & 0.816 \\
\hline AANAT & rs3744044 & 1.698 & 2.017 & 0.400 & 2.405 & $0.521-11.100$ & 0.261 & -1.358 & 2.767 & 0.624 \\
\hline$A S M T$ & rs17149149 & 1.191 & 1.490 & 0.425 & 0.962 & $0.304-3.050$ & 0.948 & 2.308 & 2.122 & 0.278 \\
\hline$A S M T$ & rs62593301 & 0.635 & 0.511 & 0.215 & 1.169 & $0.791-1.729$ & 0.434 & 0.108 & 0.700 & 0.877 \\
\hline$A S M T$ & rs28675287 & -0.312 & 0.456 & 0.495 & 1.035 & $0.728-1.470$ & 0.849 & 0.461 & 0.629 & 0.464 \\
\hline$A S M T$ & rs4521942 & 0.053 & 0.859 & 0.951 & 0.730 & $0.365-1.457$ & 0.372 & -0.138 & 1.191 & 0.908 \\
\hline$A S M T$ & rs4639690 & 0.828 & 0.446 & 0.064 & 1.414 & $1.000-1.999$ & 0.050 & -0.256 & 0.619 & 0.679 \\
\hline$A S M T$ & rs4933063 & -0.427 & 0.451 & 0.344 & 0.890 & $0.626-1.266$ & 0.518 & -0.613 & 0.618 & 0.322 \\
\hline
\end{tabular}

* corrected $\mathrm{p}$-value $<0.05$ (corrected $\mathrm{p}=0.05 * \mathrm{SNP}$ number). ${ }^{\dagger}$ nominal $\mathrm{p}$-value by logistic regression with age and sex covariates. SNP: single nucleotide polymorphism, CSM: composite scale of morningness, SE: standard error, OR: odds ratio, CI: confidence interval

id degradation, thereby reducing the synthesis of melatonin. ${ }^{60,61}$ The AANAT regulatory regions enabled the cAMP to control rapid activation and degradation switching by phosphorylating the Ser/Thr residues in the PKA/14-3-3 motifs. ${ }^{59}$

Following imputation, the rs 28936679 in the exon of AANAT also showed an association with the seasonality of BD. The rs28936679 is a nonsynonymous (missense) variant in the AANAT exon1, which participates in an amino acid substitution from alanine to threonine. Because of different chemical characteristics, such as the structure and polarity between these amino acids, the activity of AANAT might be affected by this substitution. ${ }^{62}$ This SNP was also reported to be related to de- 


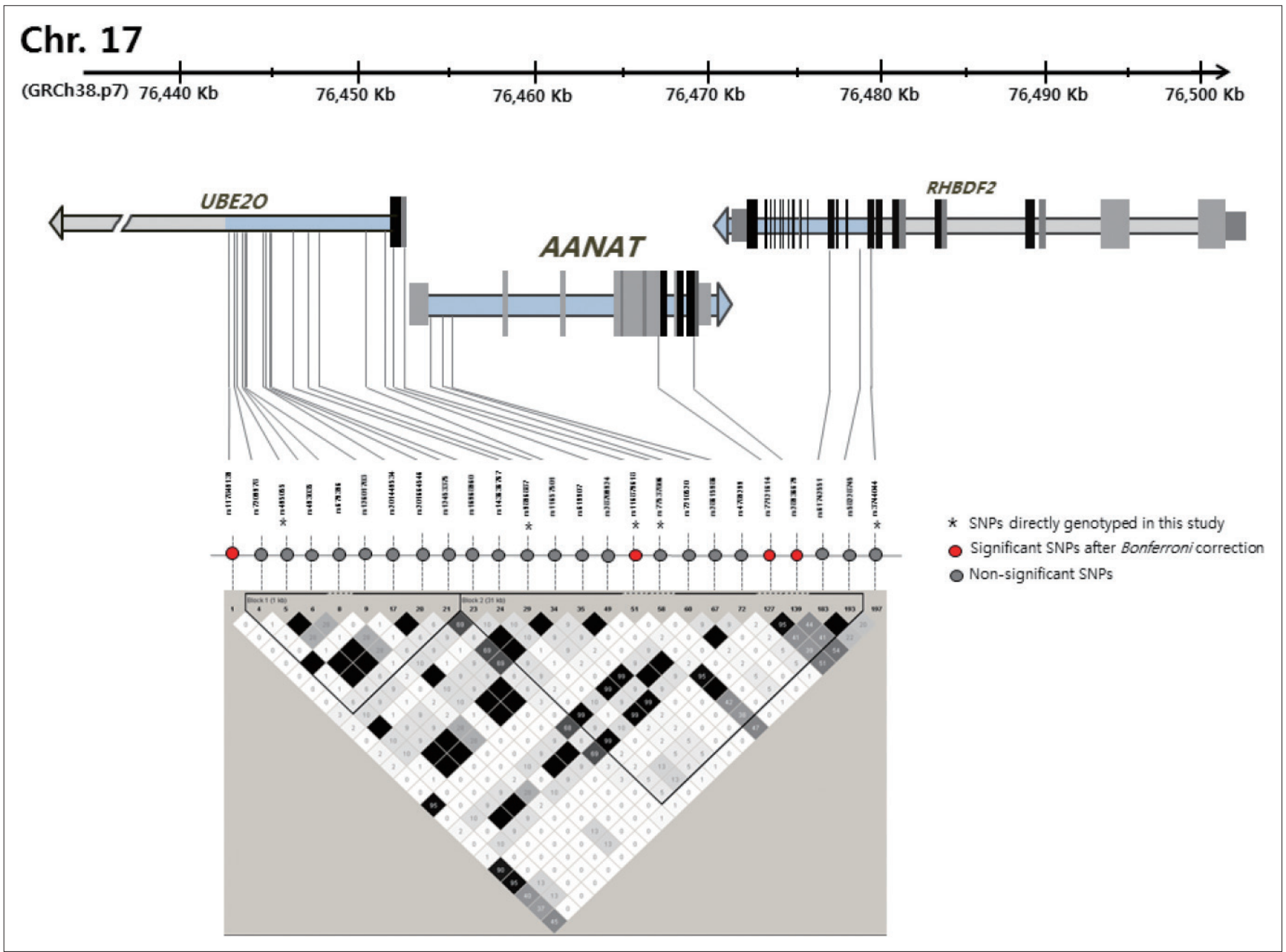

Figure 1. Scaled graphical representation of the $10 \mathrm{~Kb}$ genomic region surrounding the AANAT including association analysis results. Relative positions of SNPs directly genotyped in this study and imputed SNPs are presented. Coding exons of the genes in the regions are shown in gray, noncoding exons in black. SNPs that showed significant association with seasonality of BD are highlighted in the red circle. Bottom of diagram, there is the LD pattern of the region determined and visualized using the Haploview, and LD blocks were defined according to Gabriel criteria. ${ }^{53}$ SNP: single nucleotide polymorphism, BD: bipolar disorder, LD: linkage disequilibrium.

layed sleep phase syndrome. ${ }^{62}$

Notably in current study, the variant of AANAT was related to seasonality rather than circadian preference. A previous study based on the general population suggested that genetic variants may also contribute to the seasonality phenotype that is similar to the current study in some instances. ${ }^{63}$ The suprachiasmatic nuclei regulate seasonal rhythmicity by perceiving and encoding changes in day length and transmitting them to the pineal gland, where they regulate melatonin production. ${ }^{11,64}$ In animal studies, when entrained to a long photoperiod, the light-induced decrease in AANAT is advanced by an earlier dawn, whereas the dark-induced increase in $A A N A T$ is delayed by a later dusk. As a result, daily melatonin secretion is compressed during the summer and prolonged during the winter. ${ }^{65,66}$ Therefore, the variants of AANAT can cause change in the normal seasonal rhythm, and could affect the seasonality of BD.

This study has several limitations. First, since it did not include normal control subjects, we are not sure if the current study's findings are confined to BD or if they could be generalized in the normal population. Second, we included only four genes that directly participate in melatonin synthesis and action. Some other genes that may indirectly affect melatonergic function were not evaluated. Further studies that include more related genes and that cover genetic interactions are needed. Third, although night-shift workers were excluded from these subjects, we could not completely adjust for the effect of other social environments that could affect the individual's lifestyle and sleep-wake cycle. Finally, current study showed an association between subgroup of $\mathrm{BD}$ patient and a melatonin pathway gene, but it is unclear whether this gene causes $\mathrm{BD}$. In order to reveal this causality, future studies using methods such as Mendelian randomization are needed.

This study demonstrated an association between a genetic variation in AANAT and seasonality in BD. Further studies on the biological mechanisms of melatonin on the seasonality of $\mathrm{BD}$ are needed. 
Table 5. Association analysis of seasonality of BD with imputed SNPs in AANAT ${ }^{\dagger}$

\begin{tabular}{|c|c|c|c|c|c|c|c|c|c|}
\hline SNP & Genomic location $^{\ddagger}$ & Intragenic location & $\mathrm{M}$ & $\mathrm{m}$ & MAF & HWE & OR & $\mathrm{CI}$ & $\mathrm{p}^{\S}$ \\
\hline rs117849139 & 74440536 & 5'-near & $\mathrm{T}$ & $\mathrm{C}$ & 0.030 & 0.173 & 5.639 & $2.187-14.540$ & $<0.001^{*}$ \\
\hline rs7209978 & 74440875 & 5'-near & $\mathrm{A}$ & G & 0.094 & 0.227 & 0.963 & $0.549-1.689$ & 0.895 \\
\hline rs495055 & 74440890 & 5'-near & $\mathrm{A}$ & G & 0.082 & 1.000 & 0.674 & $0.345-1.318$ & 0.249 \\
\hline rs493035 & 74441157 & 5'-near & $\mathrm{C}$ & $\mathrm{T}$ & 0.082 & 1.000 & 0.674 & $0.345-1.318$ & 0.249 \\
\hline rs679396 & 74441310 & 5'-near & $\mathrm{T}$ & $\mathrm{C}$ & 0.021 & 0.310 & 0.478 & $0.125-1.829$ & 0.281 \\
\hline rs 12601703 & 74441316 & 5 'near & $\mathrm{T}$ & $\mathrm{C}$ & 0.094 & 0.227 & 0.963 & $0.549-1.689$ & 0.895 \\
\hline rs201449534 & 74442545 & 5'-near & - & $\mathrm{C}$ & 0.082 & 1.000 & 0.674 & $0.345-1.318$ & 0.249 \\
\hline rs201664546 & 74442551 & 5'-near & A & $\mathrm{C}$ & 0.082 & 1.000 & 0.674 & $0.345-1.318$ & 0.249 \\
\hline rs12453375 & 74442650 & 5 'near & G & A & 0.397 & 0.480 & 1.256 & $0.905-1.744$ & 0.172 \\
\hline rs 16968960 & 74442942 & 5 'near & A & G & 0.491 & 0.200 & 1.242 & $0.894-1.726$ & 0.196 \\
\hline rs143636767 & 74442979 & 5 'near & $\begin{array}{l}\text { TGTGTT } \\
\text { TTTGAC }\end{array}$ & - & 0.094 & 0.228 & 0.958 & $0.546-1.681$ & 0.882 \\
\hline rs9896887 & 74444059 & 5'-near & G & A & 0.492 & 0.201 & 1.242 & $0.894-1.726$ & 0.196 \\
\hline rs11657501 & 74445051 & 5'-near & A & G & 0.489 & 0.176 & 1.242 & $0.894-1.726$ & 0.196 \\
\hline rs619907 & 74445776 & 5 '-near & G & A & 0.082 & 1.000 & 0.674 & $0.345-1.318$ & 0.249 \\
\hline rs28709924 & 74448395 & 5 'near & $\mathrm{C}$ & G & 0.082 & 1.000 & 0.674 & $0.345-1.318$ & 0.249 \\
\hline rs116879618 & 74448849 & 5 'near & $\mathrm{C}$ & $\mathrm{T}$ & 0.031 & 0.193 & 4.918 & $2.001-12.090$ & $<0.001^{*}$ \\
\hline rs77537806 & 74449204 & 5 'near & G & $\mathrm{T}$ & 0.015 & 0.171 & 2.331 & $0.772-7.035$ & 0.133 \\
\hline rs7210520 & 74450040 & Intron & $\mathrm{C}$ & $\mathrm{T}$ & 0.491 & 0.248 & 1.232 & $0.886-1.713$ & 0.215 \\
\hline rs 28615986 & 74451270 & Intron & G & $\mathrm{C}$ & 0.082 & 0.824 & 0.690 & $0.352-1.353$ & 0.280 \\
\hline rs4789299 & 74452010 & Intron & G & A & 0.488 & 0.247 & 1.250 & $0.898-1.741$ & 0.187 \\
\hline rs77121614 & 74464146 & Intron & $\mathrm{C}$ & $\mathrm{T}$ & 0.028 & 0.489 & 5.282 & $2.037-13.700$ & $<0.001^{*}$ \\
\hline rs28936679 & 74465813 & Exon (n-syn) & G & A & 0.027 & 0.458 & 6.411 & $2.306-17.830$ & $<0.001^{*}$ \\
\hline rs61742551 & 74472998 & Exon (syn) & G & A & 0.060 & 0.535 & 2.588 & $1.370-4.887$ & 0.003 \\
\hline rs58228745 & 74474330 & Intron & $\mathrm{C}$ & $\mathrm{T}$ & 0.061 & 0.554 & 2.568 & $1.377-4.788$ & 0.003 \\
\hline rs3744044 & 74475014 & 3'-near & $\mathrm{C}$ & $\mathrm{T}$ & 0.012 & 0.114 & 2.819 & $0.736-10.800$ & 0.131 \\
\hline
\end{tabular}

*corrected $\mathrm{p}$-value $<0.05$ (corrected $\mathrm{p}=0.05^{*} \mathrm{SNP}$ number), timputation using the reference panel of the EAS data from the $1000 \mathrm{Genomes}$ Project Integrated Phase 3 Release, ‡Genomic Location was released from Human Feb. 2009 (GRCh37/hg19) Assembly, §nominal p-value by logistic regression with age and sex covariates. SNP: single nucleotide polymorphism, M: major allele, m: minor allele, MAF: minor allele frequency, HWE: p-value of the Hardy-Weinberg equilibrium test, UTR: untranslated region, OR: odds ratio, CI: confidence interval, n-syn: nonsynonymous, syn: synonymous

\section{Supplementary Materials}

The online-only Data Supplement is available with this article at https://doi.org/10.30773/pi.2020.0436.

\section{Acknowledgments}

This work was supported by the Original Technology Research Program for Brain Science through the National Research Foundation of Korea (NRF) funded by the Ministry of Science and ICT (MSIT) [No. 2019M3 C7A1030624]

\section{Conflicts of Interest}

The authors have no potential conflicts of interest to disclose.

\section{Author Contributions}

Conceptualization: So Yung Yang, Kyung Sue Hong, Ji Hyun Baek. Data curation: Kyung Sue Hong, Youngah Cho, Yujin Choi, Kyooseob Ha, Ji Hyun Baek. Formal analysis: So Yung Yang, Yongkang Kim, Taesung Park. Funding acquisition: Kyung Sue Hong. Investigation: Eun-Young Cho.
Methodology: So Yung Yang, Kyung Sue Hong. Project administration: Kyung Sue Hong. Software: Yongkang Kim, Taesung Park. Supervision: Kyung Sue Hong. Validation: Kyung Sue Hong, Ji Hyun Baek. Visualization: So Yung Yang, Eun-Yung Cho. Writing-original draft: So Yung Yang, Kyung Sue Hong. Writing—review \& editing: So Yung Yang, Kyung Sue Hong, Ji Hyun Baek.

\section{ORCID iDs}

So Yung Yang

Kyung Sue Hong

Youngah Cho

Eun-Young Cho

Yujin Choi

Yongkang Kim

Taesung Park

Kyooseob Ha

Ji Hyun Baek https://orcid.org/0000-0001-7310-6795 https://orcid.org/0000-0002-1250-6151 https://orcid.org/0000-0003-2548-0293 https://orcid.org/0000-0002-2989-8307 https://orcid.org/0000-0002-6112-5267 https://orcid.org/0000-0002-8837-2620 https://orcid.org/0000-0002-8294-590X https://orcid.org/0000-0001-5035-2950 https://orcid.org/0000-0002-1366-0396 


\section{REFERENCES}

1. American Psychiatric Association. Diagnostic and Statistical Manual of Mental Disorders: DSM-5. 5th ed. Arlington, VA: American Psychaitric Association;2013.

2. Smoller JW, Finn CT. Family, twin, and adoption studies of bipolar disorder. Am J Med Genet C Semin Med Genet 2003;123C:48-58.

3. Craddock N, Sklar P. Genetics of bipolar disorder. Lancet 2013;381: 1654-1662.

4. Charney A, Ruderfer D, Stahl E, Moran J, Chambert K, Belliveau R, et al. Evidence for genetic heterogeneity between clinical subtypes of bipolar disorder. Transl Psychiatry 2017;7:e993.

5. International Schizophrenia Consortium; Purcell SM, Wray NR, Stone JL, Visscher PM, O’Donovan MC, et al. Common polygenic variation contributes to risk of schizophrenia and bipolar disorder. Nature 2009; 460:748-752.

6. Leboyer M, Bellivier F, Nosten-Bertrand M, Jouvent R, Pauls D, Mallet J, et al. Psychiatric genetics: search for phenotypes. Trends Neurosci 1998;21:102-105.

7. Hasler G, Drevets WC, Gould TD, Gottesman II, Manji HK. Toward constructing an endophenotype strategy for bipolar disorders. Biol Psychiatry 2006;60:93-105.

8. Bipolar Disorder and Schizophrenia Working Group of the Psychiatric Genomics Consortium. Genomic Dissection of Bipolar Disorder and Schizophrenia, Including 28 Subphenotypes. Cell 2018;173:1705-1715. e16.

9. DeCoursey PJ, Krulas JR. Behavior of SCN-lesioned chipmunks in natural habitat: a pilot study. J Biol Rhythms 1998;13:229-244.

10. Konopka RJ, Benzer S. Clock mutants of Drosophila melanogaster. Proc Natl Acad Sci U S A 1971;68:2112-2116.

11. Coomans CP, Ramkisoensing A, Meijer JH. The suprachiasmatic nuclei as a seasonal clock. Front Neuroendocrinol 2015;37:29-42.

12. Wood C, Magnello M. Diurnal changes in perceptions of energy and mood. J R Soc Med 1992; 85:191-194.

13. Grimaldi S, Partonen T, Haukka J, Aromaa A, Lönnqvist J. Seasonal vegetative and affective symptoms in the Finnish general population: testing the dual vulnerability and latitude effect hypotheses. Nord J Psychiatry 2009;63:397-404.

14. Lall GS, Atkinson LA, Corlett S, Broadbridge P, Bonsall DR. Circadian entrainment and its role in depression: a mechanistic review. J Neural Transm (Vienna) 2012;119:1085-1096.

15. Lanfumey L, Mongeau R, Hamon M. Biological rhythms and melatonin in mood disorders and their treatments. Pharmacol Ther 2013; 138:176-184.

16. Gonzalez R. The relationship between bipolar disorder and biological rhythms. J Clin Psychiatry 2014;75:e323-e331.

17. Geoffroy PA, Bellivier F, Scott J, Etain B. Seasonality and bipolar disorder: a systematic review, from admission rates to seasonality of symptoms. J Affect Disord 2014;168:210-223.

18. Melo MC, Abreu RL, Linhares Neto VB, de Bruin PF, de Bruin VM. Chronotype and circadian rhythm in bipolar disorder: a systematic review. Sleep Med Rev 2017;34:46-58.

19. Shin K, Schaffer A, Levitt AJ, Boyle MH. Seasonality in a community sample of bipolar, unipolar and control subjects. J Affect Disord 2005; 86:19-25.

20. Shand AJ, Scott NW, Anderson SM, Eagles JM. The seasonality of bipolar affective disorder: comparison with a primary care sample using the Seasonal Pattern Assessment Questionnaire. J Affect Disord 2011; 132:289-292.

21. Myers D, Davies P. The seasonal incidence of mania and its relationship to climatic variables. Psychol Med 1978;8:433-440.

22. Lee HJ, Kim L, Joe SH, Suh KY. Effects of season and climate on the first manic episode of bipolar affective disorder in Korea. Psychiatry Res 2002;113:151-159.

23. Yang AC, Yang CH, Hong CJ, Liou YJ, Shia BC, Peng CK, et al. Effects of age, sex, index admission, and predominant polarity on the seasonality of acute admissions for bipolar disorder: a population-based study. Chronobiol Int 2013;30:478-485.

24. Moreira J, Geoffroy PA. Lithium and bipolar disorder: impacts from molecular to behavioural circadian rhythms. Chronobiol Int 2016;33: 351-373.

25. Hallam KT, Olver JS, Norman TR. Effect of sodium valproate on nocturnal melatonin sensitivity to light in healthy volunteers. Neuropsychopharmacology 2005;30:1400-1404.

26. Geoffroy PA, Bellivier F, Scott J, Boudebesse C, Lajnef M, Gard S, et al. Bipolar disorder with seasonal pattern: clinical characteristics and gender influences. Chronobiol Int 2013;30:1101-1107.

27. Baek JH, Kim JS, Kim MJ, Ryu S, Lee K, Ha K, et al. Lifetime characteristics of evening-preference and irregular bed-rise time are associated with lifetime seasonal variation of mood and behavior: comparison between individuals with bipolar disorder and healthy controls. Behav Sleep Med 2016;14:155-168.

28. Romo-Nava F, Blom TJ, Cuellar-Barboza AB, Winham SJ, Colby CL, Nunez NA, et al. Evening chronotype as a discrete clinical subphenotype in bipolar disorder. J Affect Disord 2020;266:556-562.

29. Choi J, Baek JH, Noh J, Kim JS, Choi JS, Ha K, et al. Association of seasonality and premenstrual symptoms in bipolar I and bipolar II disorders. J Affect Disord 2011;129:313-316.

30. Klein DC, Berg GR, Weller J. Melatonin synthesis: adenosine 3', 5' -monophosphate and norepinephrine stimulate $\mathrm{N}$-acetyltransferase. Science 1970;168:979-980.

31. Foulkes NS, Whitmore D, Sassone-Corsi P. Rhythmic transcription: the molecular basis of circadian melatonin synthesis. Biol Cell 1997;89: 487-494.

32. Dubocovich ML, Markowska M. Functional MT 1 and MT 2 melatonin receptors in mammals. Endocrine 2005;27:101-110.

33. Etain B, Dumaine A, Bellivier F, Pagan C, Francelle L, Goubran-Botros $\mathrm{H}$, et al. Genetic and functional abnormalities of the melatonin biosynthesis pathway in patients with bipolar disorder. Hum Mol Genet 2012;21:4030-4037.

34. Soria V, Martínez-Amorós È, Escaramís G, Valero J, Crespo JM, Gutiérrez-Zotes A, et al. Resequencing and association analysis of arylalkylamine $\mathrm{N}$-acetyltransferase (AANAT) gene and its contribution to major depression susceptibility. J Pineal Res 2010;49:35-44.

35. Mansour HA, Talkowski ME, Wood J, Chowdari KV, McClain L, Prasad K, et al. Association study of 21 circadian genes with bipolar I disorder, schizoaffective disorder, and schizophrenia. Bipolar Disord 2009;11:701-710.

36. Kripke DF, Nievergelt CM, Tranah GJ, Murray SS, McCarthy MJ, Rex $\mathrm{KM}$, et al. Polymorphisms in melatonin synthesis pathways: possible influences on depression. J Circadian Rhythms 2011;9:8.

37. Geoffroy PA, Lajnef M, Bellivier F, Jamain S, Gard S, Kahn JP, et al. Genetic association study of circadian genes with seasonal pattern in bipolar disorders. Sci Rep 2015;5:1-8.

38. Geoffroy PA, Boudebesse C, Henrion A, Jamain S, Henry C, Leboyer $\mathrm{M}$, et al. An ASMT variant associated with bipolar disorder influences sleep and circadian rhythms: a pilot study. Genes Brain Behav 2014;13: 299-304.

39. Han O, Hong J. SCID-I; Structured Clinical Interview for Axis I Disorder of DSM-IV. Seoul, Korea: Hana Medical; 2000

40. Joo EJ, Joo YH, Hong JP, Hwang S, Maeng SJ, Han JH, et al. Korean version of the diagnostic interview for genetic studies: validity and reliability. Compr Psychiatry 2004;45:225-229.

41. Grandin LD, Alloy LB, Abramson LY. The social zeitgeber theory, circadian rhythms, and mood disorders: review and evaluation. Clin Psychol Rev 2006;26:679-694.

42. Smith CS, Reilly C, Midkiff K. Evaluation of three circadian rhythm questionnaires with suggestions for an improved measure of morningness. J Appl Psychol 1989;74:728-738.

43. Yoon JS, Shin SM, Kook SH, Lee HY. A preliminary study on the Ko- 
rean translation of composite scale (KtCS) to measure morningness: eveningness. J Korean Neuropsychiatr Assoc 1997;36:122-134.

44. Rosenthal NE, Sack DA, Gillin JC, Lewy AJ, Goodwin FK, Davenport $\mathrm{Y}$, et al. Seasonal affective disorder: a description of the syndrome and preliminary findings with light therapy. Arch Gen Psychiatry 1984;41: 72-80.

45. Baek JH, Kim JS, Huh I, Lee K, Park JH, Park T, et al. Prevalence, behavioral manifestations and associated individual and climatic factors of seasonality in the Korean general population. Compr Psychiatry 2015;57:148-154.

46. Kasper S, Wehr TA, Bartko JJ, Gaist PA, Rosenthal NE. Epidemiological findings of seasonal changes in mood and behavior: a telephone survey of Montgomery County, Maryland. Arch Gen Psychiatry 1989; 46:823-833.

47. Magnusson A, Stefansson JG. Prevalence of seasonal affective disorder in Iceland. Arch Gen Psychiatry 1993;50:941-946.

48. Flaquer A, Jamra RA, Etterer K, Díaz GO, Rivas F, Rietschel M, et al. A new susceptibility locus for bipolar affective disorder in PAR1 on Xp22. 3/Yp11. 3. Am J Med Genet B Neuropsychiatr Genet 2010;153B:11101114.

49. Chang CC, Chow CC, Tellier LC, Vattikuti S, Purcell SM, Lee JJ. Second-generation PLINK: rising to the challenge of larger and richer datasets. Gigascience 2015;4:7.

50. Delaneau O, Marchini J, Zagury JF. A linear complexity phasing method for thousands of genomes. Nat Methods 2012;9:179-181.

51. Howie BN, Donnelly P, Marchini J. A flexible and accurate genotype imputation method for the next generation of genome-wide association studies. PLoS Genet 2009;5:e1000529.

52. Barrett JC, Fry B, Maller J, Daly MJ. Haploview: analysis and visualization of LD and haplotype maps. Bioinformatics 2005;21:263-265.

53. Gabriel SB, Schaffner SF, Nguyen H, Moore JM, Roy J, Blumenstiel B, et al. The structure of haplotype blocks in the human genome. Science 2002;296:2225-2229.

54. Lewy AJ, Nurnberger JI, Wehr TA, Pack D, Becker LE, Powell RL, et al. Supersensitivity to light: possible trait marker for manic-depressive illness. Am J Psychiatry 1985;142:725-727.

55. Nurnberger JI, Adkins S, Lahiri DK, Mayeda A, Hu K, Lewy A, et al. Melatonin suppression by light in euthymic bipolar and unipolar pa- tients. Arch Gen Psychiatry 2000;57:572-579.

56. Dick DM, Foroud T, Flury L, Bowman ES, Miller MJ, Rau NL, et al. Genomewide linkage analyses of bipolar disorder: a new sample of 250 pedigrees from the National Institute of Mental Health Genetics Initiative. Am J Hum Genet 2003;73:107-114.

57. Zandi PP, Badner J, Steele J, Willour V, Miao K, Mackinnon DF, et al. Genome-wide linkage scan of 98 bipolar pedigrees and analysis of clinical covariates. Mol Psychiatry 2007;12:630-639.

58. Ewald H, Wikman F, Teruel B, Buttenschøn HN, Torralba M, Als TD, et al. A genome-wide search for risk genes using homozygosity mapping and microarrays with 1,494 single-nucleotide polymorphisms in 22 eastern Cuban families with bipolar disorder. Am J Med Genet B Neuropsychiatr Genet 2005;133B:25-30.

59. Klein DC. Arylalkylamine N-acetyltransferase:"the Timezyme". J Biol Chem 2007;282:4233-4237.

60. Weitzman E, Weinberg U, D'Eletto R, Lynch H, Wurtman R, Czeisler C, et al. Studies of the 24 hour rhythm of melatonin in man. J Neural Transm Suppl 1978;(13):325-337.

61. Waldhauser F, Waldhauser M, Lieberman HR, Deng MH, Lynch HJ, Wurtman RJ. Bioavailability of oral melatonin in humans. Neuroendocrinology 1984;39:307-313.

62. Hohjoh H, Takasu M, Shishikura K, Takahashi Y, Honda Y, Tokunaga K. Significant association of the arylalkylamine $\mathrm{N}$-acetyltransferase (AA-NAT) gene with delayed sleep phase syndrome. Neurogenetics 2003;4:151-153.

63. Yang SY, Baek JH, Cho Y, Cho EY, Choi Y, Kim Y, et al. Effects of genetic variants of ST8SIA2 and NCAM1 genes on seasonal mood changes and circadian preference in the general population. Chronobiol Int 2018; 35:405-415.

64. VanderLeest HT, Houben T, Michel S, Deboer T, Albus H, Vansteensel MJ, et al. Seasonal encoding by the circadian pacemaker of the SCN. Curr Biol 2007;17:468-473.

65. Illnerová H, Vaněček J. Pineal rhythm in N-acetyltransferase activity in rats under different artificial photoperiods and in natural daylight in the course of a year. Neuroendocrinology 1980;31:321-326.

66. Illnerova H, Vaněcek J. Regulation of the circadian rhythm in pineal melatonin production. Physiol Bohemoslov 1985;34(Suppl):57-61. 
Supplementary Table 1. Results of association test of SNPS in melatonin pathway genes with bipolar patients's seasonality and circadian
preference

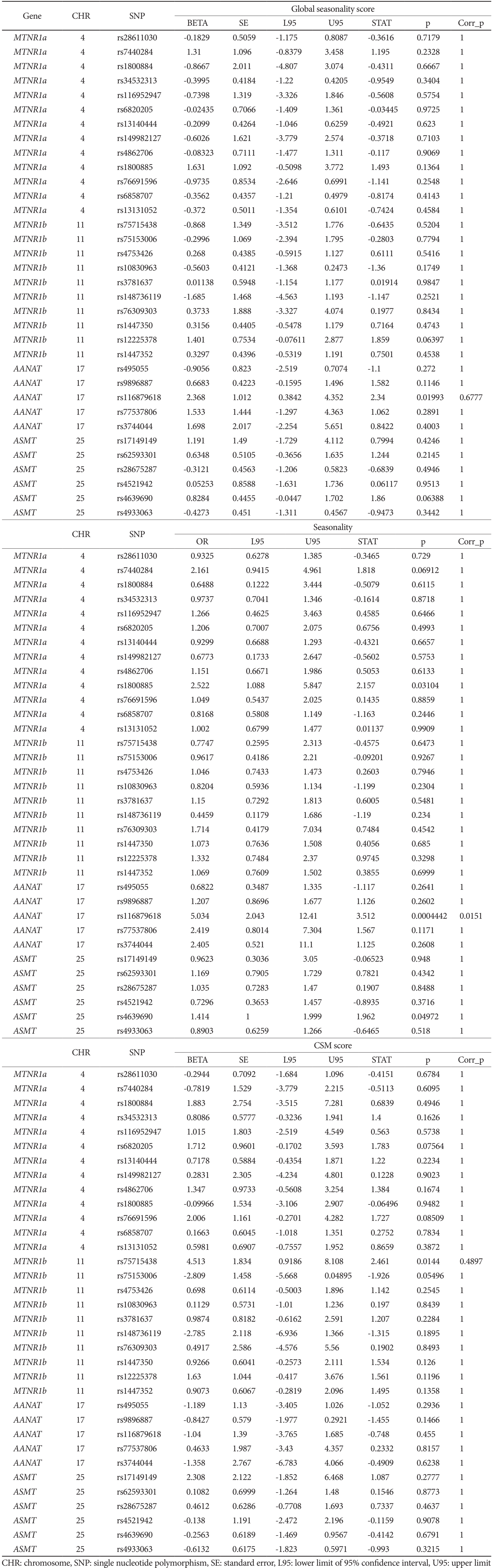


Supplementary Table 2. Result of association test of seasonality of BD with imputed SNPs in AANAT

\begin{tabular}{|c|c|c|c|c|}
\hline \multirow{2}{*}{ SNP } & \multicolumn{4}{|c|}{ Additive } \\
\hline & OR & CI & $\mathrm{p}$ & Corr_p \\
\hline rs117849139 & 5.639 & $2.187-14.54$ & 0.000345 & 0.008634 \\
\hline rs7209978 & 0.9629 & $0.549-1.689$ & 0.8952 & 1 \\
\hline rs495055 & 0.6739 & $0.3445-1.318$ & 0.249 & 1 \\
\hline rs493035 & 0.6739 & $0.3445-1.318$ & 0.249 & 1 \\
\hline rs679396 & 0.4779 & $0.1249-1.829$ & 0.2808 & 1 \\
\hline rs12601703 & 0.9629 & $0.549-1.689$ & 0.8952 & 1 \\
\hline rs201449534 & 0.6739 & $0.3445-1.318$ & 0.249 & 1 \\
\hline rs201664546 & 0.6739 & $0.3445-1.318$ & 0.249 & 1 \\
\hline rs12453375 & 1.256 & $0.9054-1.744$ & 0.1721 & 1 \\
\hline rs16968960 & 1.242 & $0.8941-1.726$ & 0.196 & 1 \\
\hline rs143636767 & 0.9583 & $0.5464-1.681$ & 0.8819 & 1 \\
\hline rs9896887 & 1.242 & $0.8941-1.726$ & 0.196 & 1 \\
\hline rs11657501 & 1.242 & $0.8944-1.726$ & 0.1955 & 1 \\
\hline rs619907 & 0.6739 & $0.3445-1.318$ & 0.249 & 1 \\
\hline rs28709924 & 0.6739 & $0.3445-1.318$ & 0.249 & 1 \\
\hline rs116879618 & 4.918 & $2.001-12.09$ & 0.000517 & 0.01292 \\
\hline rs77537806 & 2.331 & $0.7721-7.035$ & 0.1333 & 1 \\
\hline rs7210520 & 1.232 & $0.886-1.713$ & 0.2148 & 1 \\
\hline rs28615986 & 0.6901 & $0.352-1.353$ & 0.2803 & 1 \\
\hline rs4789299 & 1.25 & $0.8975-1.741$ & 0.1867 & 1 \\
\hline rs77121614 & 5.282 & $2.037-13.7$ & 0.000619 & 0.01547 \\
\hline rs28936679 & 6.411 & $2.306-17.83$ & 0.00037 & 0.009236 \\
\hline rs61742551 & 2.588 & $1.37-4.887$ & 0.003377 & 0.08443 \\
\hline rs58228745 & 2.568 & $1.377-4.788$ & 0.003017 & 0.07542 \\
\hline rs3744044 & 2.819 & $0.7357-10.8$ & 0.1305 & 1 \\
\hline
\end{tabular}

BD: bipolar disorder, SNP: single nucleotide polymorphism, OR: odds ratio, CI: confidence interval, Corr_p: corrected p-value 